\title{
Mono-oligoclonal immunoglobulin abnormalities in diabetic patients after kidney transplantation: influence of simultaneous pancreas graft
}

\author{
M. Bernardi ${ }^{1}$, E. La Rocca ${ }^{1}$, R. Castoldi ${ }^{2}$, V. Di Carlo ${ }^{2}$, R. Caldara ${ }^{1}$, S. Furiani ${ }^{1}$, D. Giudici ${ }^{3}$, G. Pozza ${ }^{1}$, A. Secchi ${ }^{1}$ \\ ${ }^{1}$ Department of Internal Medicine, San Raffaele Scientific Institute, University of Milan, Milan, Italy \\ ${ }^{2}$ Department of General Surgery, San Raffaele Scientific Institute, University of Milan, Milan, Italy \\ ${ }^{3}$ Department of Anesthesiology, San Raffaele Scientific Institute, University of Milan, Milan, Italy
}

Summary Monoclonal components (MC) are detected in as high as $30 \%$ of renal transplant recipients. Our aim was to evaluate the incidence, relevance and consequence of monoclonal components in patients with Type I (insulin-dependent) diabetes who received kidney $(n=22)$, kidney and whole pancreas $(n=41)$, kidney and segmental pancreas $(n=24)$ and kidney and islets $(n=12)$ transplants. Immunosuppression was based on prophylactic anti-lymphocyte globulins, corticosteroids, azathioprine and cyclosporin in all patients; acute rejection was treated with steroids or anti-lymphocyte monoclonal immunoglobulin therapy (OKT3) or both. Serum immunofixation was carried out in all patients before transplantation and then after at 6 months and then yearly. Monoclonal components were detected in 81 of 99 patients $(82 \%) ; 52$ patients $(52 \%)$ developed them within 6 months of transplantation, $15(15 \%)$ between 6 and 12 months, with a peak prevalence at 1 year post-transplant (58\%) and a decrease thereafter (10\% at 9 years). Kidney recipients showed a lower incidence of monoclonal components when compared with those who received kidneys and segmental pancreases and those who received kidneys and whole pancreases. Monoclonal components were more often detected in patients who had previously experienced an acute renal rejection. Cytomegalovirus infection and acute rejection occurring in the same patient further increased the risk of developing monoclonal components, the development of which did not correlate with OKT3 treatment. A Post-transplant lymphoproliferative disorder was developed by two patients $(2 \%)$, one with 5 and the other with 6 monoclonal components. In conclusion, diabetic patients receiving kidney and/or Pancreas transplantation, experiencing both cytomegalovirus infection and acute rejection, are at greatest risk of developing monoclonal components but they appear to be benign and transient; multiple band detection is a marker for the subsequent development of post-transplant lymphoprolifertive disorder. [Diabetologia (1998) 41: 1176-1179]

Keywords Pancreas transplantation, kidney transplantation, monoclonal immunoglobulins, immunosuppression
Received: 5 January 1998 and in revised form: 28 April 1998

Corresponding author: A. Secchi, MD, San Raffaele Scientific Institute, University of Milan, Via Olgettina 60, I-20132 Milano, Italy

Abbreviations: MC; monoclonal components; PTLD, posttransplant lymphoproliferative disorder; OKT3, ant-lymphocyte monoclonal immunoglobin therapy; EBV, Epstein-Barr Virus; CMV, cytomegalovirus; ATG, antithymocyte globulin serum. ALG, Antilymphocyte globulin serum
Kidney transplantation is the preferable treatment in Type I (insulin-dependent) diabetic uremic patients as life expectancy greatly improves with a successful kidney transplant and withdrawal from haemodialysis is possible [1]. In these patients a simultaneous transplant of the pancreas has a positive effect on patient survival and quality of life because metabolic control and neuropathy are improved [1-3].

A higher incidence of graft rejection has been described after simultaneous kidney-pancreas transplantation than after kidney transplant alone [4], re- 
quiring a cumulative higher degree of immunosuppression, with an increased risk of short and longterm complications, such as infections or malignancies or both [5-9]. Nevertheless preliminary studies have not shown a difference between the two groups of patients in terms of incidence of malignancies [10].

A strikingly high incidence of post-transplant lymphoproliferative disorder (PTLD) has been observed after cyclosporin treatment, and anti-lymphocyte monoclonal immunoglobulin therapy (OKT3), most of which are non-Hodgkin lymphomas (NHL) of beta-cell lineage, occurring early after transplantation and in some cases regressing after reduction or withdrawal of immunosuppression $[6,11]$.

The incidence of monoclonal components (MC) in the general population is $0.1-1.25 \%$, increasing with age; some studies report a peak prevalence of $3 \%$ in people older than 70 years $[12,13]$. They are associated with a variety of conditions, including neoplasms of the lymphoproliferative system, so-called gammopathies of undefined significance (MGUS), solid neoplasms and non-tumorous diseases (e.g. autoimmune diseases). In some cases they disappear when patients recover from the underlying disease, but they are more often persistent in chronic disorders or may finally transform into multiple myeloma. Immunoglobulin abnormalities are a frequent finding after renal transplantation. They are detected in as high as $30 \%$ of renal transplant recipients, differing from those found normally in humans because they are more often multiple and are usually transient $[14,15]$. Such MC, reflecting the expansion of betacell clones, may precede a PTLD: they necessitate careful evaluation and follow-up.

The aim of our study was to evaluate MC incidence, risk factors for MC development and MC significance in patients with Type I diabetes who underwent kidney, kidney and pancreas, and kidney and islets transplants.

\section{Subjects and methods}

Patients. We studied the 99 patients with Type I diabetes who underwent a renal transplantation at our institute between July 1985 and November 1995. Of the patients 65 received a simultaneous kidney and pancreas transplant comprising 41 whole pancreas transplants with bladder-derived exocrine secretions and 24 segmental, neoprene-injected, pancreas transplants. Of the remaining 34 patients 12 received simultaneous islets-kidney transplantation and 22 only a kidney.

Immunosuppression. Prophylactic immunosuppression was based on a sequential quadruple regimen in all patients: antilymphocyte serum (ALG: 4250 lymphocytotoxic units . $\mathrm{kg}^{-1} \cdot$ day $^{-1}$ for the first 10 days between July 1985 and March 1994 or antithymocyte globulin serum: ATG: $125 \mathrm{mg} /$ day for the first 10 days after June 1994); cyclosporin, $7 \mathrm{mg}$. $\mathrm{kg}^{-1} \cdot$ day $^{-1}$ adjusted according to whole blood levels trough concentration of 150-250 ng/ml (EIA, monoclonal); azathio- prine, $150 \mathrm{mg} /$ day then tapered according to leucocyte cell count; steroids, $1 \mathrm{mg} \cdot \mathrm{kg}^{-1} \cdot$ day $^{-1}$ between $1985-1989$ or $0.25 \mathrm{mg} \cdot \mathrm{kg}^{-1} \cdot$ day $^{-1}$ since 1990 , then tapered to $10 \mathrm{mg} /$ day in both cases. Anti-acute rejection treatment was based on three to five intravenous boluses $(500 \mathrm{mg})$ of methylprednisolone; OKT3 was given for steroid-resistant rejection episodes.

Methods. All sera were investigated using the DAKO immunofixation kit (DAKO A/S, Glostrup, Denmark) with minor personal modifications. The sera were diluted with $0.9 \%$ saline to obtain a 1 gram per litre concentration of immunoglobulins, then were applied to the agarose-gel; electrophoresis was done in an electric field of $100 \mathrm{~V}$ for $25 \mathrm{~min}$ in a tri-barbiturate buffer. Anti-sera against human immunoglobulin heavy and light chains (anti-g, m, a, k, l) were applied to the gel after electrophoresis; after a $15 \mathrm{~min}$ incubation in a moist chamber, unfixed proteins were removed by rinsing in $0.9 \%$ saline. The gel was then dried and stained with black-amid; protein bands were visually evaluated.

Epstein-Barr virus (EBV) and cytomegalovirus (CMV) serology (IgG and IgM: ELFA, Biomerieux, Lyon, France) was evaluated at regular intervals before and after transplantation. We defined "seroconversion" as the development of IgG in a previous negative patient and "reactivation" as the development of IgM in an IgG positive patient.

Research plan. Immunofixation was evaluated in all patients before transplantation and after transplantation weekly during the first 2 months, at 6 months and then yearly. Incidence of rejection episodes, CMV infections and OKT3 treatment was calculated for each patient. Data were compared by means of the chi-square test, with Yates correction for continuity.

\section{Results}

Clinical characteristics of patients are reported in Table 1 . The rate of kidney rejections was higher in patients receiving kidney-pancreas than in patients receiving kidney transplant alone. The other variables were similar in the four groups of patients. All but one patient had a negative immunofixation for $\mathrm{MC}$ before transplantation. We detected MC in 81 out of 99 patients $(82 \%)$ after transplantation. All MC were quantitatively less than 1 gram per litre. At the onset $51 \%$ of MC were kappa, $28 \%$ were lambda and $21 \%$ were kappa-lambda. Patients receiving kidney and pancreas transplantation showed insignificantly higher MC incidence than patients receiving only kidney transplants (kidney and pancreas $90 \%$, kidney and segmental pancreas $83 \%$, kidney $73 \%$, kidney and islets $67 \%$ ). Within 6 months of transplantation 52 patients $(52 \%)$ developed MC, $15(15 \%)$ between 6 and 12 months, with a peak prevalence at 1 year posttransplantation $(58 \%)$ and a decrease thereafter (10\% at 9 years). No difference of MC prevalence was observed among the four groups of patients (Table 2). The increase of MC prevalence at 9 years $(10 \%)$ is due to recurrence at 8 years of 3 bands MC in one patient affected by renal failure. This patient returned to dialysis at 10 years, with a reduction of MC from 3 to 1 band. The mean onset of MC was at 
Table 1. Clinical characteristics of patients, according to the type of transplant. Data are reported as mean \pm SD

\begin{tabular}{llllll}
\hline & \multicolumn{3}{l}{ Kidney } & & \\
\cline { 2 - 6 } & + whole pancreas & + segmental pancreas & alone & + islets & overall \\
\hline$n$ & 41 & 24 & 22 & 12 & 99 \\
Age & $39 \pm 6$ & $39 \pm 6$ & $43 \pm 8$ & $39 \pm 5$ & $40 \pm 7$ \\
men/women & $23 / 18$ & $12 / 24$ & $13 / 9$ & $7 / 12$ & $55 / 44$ \\
Type I diabetes (yrs) & $25 \pm 4$ & $25 \pm 4$ & $21 \pm 6$ & $25 \pm 6$ & $24 \pm 5$ \\
Acute rejection & $31(76 \%)$ & $13(54 \%)$ & $6(27 \%)$ & $4(33 \%)$ & $54(55 \%)$ \\
CMV infection & $23(56 \%)$ & $11(46 \%)$ & $14(64 \%)$ & $4(33 \%)$ & $52(53 \%)$ \\
\hline
\end{tabular}

Table 2. Prevalence of $\mathrm{MC}$ in diabetic patients who underwent transplantation. Data is reported as absolute values (N) and prevalence $(\%)$

\begin{tabular}{|c|c|c|c|c|c|}
\hline & Kidney & & & & \\
\hline & + whole pancreas & + segmental pancreas & alone & + islets & overall \\
\hline Pre-transplant & 0/41 (0\%) & 1/24 (4\%) & $0 / 22(0 \%)$ & 0/12 (0\%) & 1/99 (1 \%) \\
\hline Discharge & $18 / 41(44 \%)$ & $7 / 24(29 \%)$ & $1 / 22(4 \%)$ & $1 / 12(8 \%)$ & $27 / 99(27 \%)$ \\
\hline+6 months & $19 / 39(49 \%)$ & $13 / 24(54 \%)$ & $9 / 22(41 \%)$ & $5 / 12(41 \%)$ & 46/97 (47\%) \\
\hline+1 year & $20 / 36(55 \%)$ & $15 / 24(62 \%)$ & $12 / 21(57 \%)$ & $7 / 12(58 \%)$ & $54 / 93(58 \%)$ \\
\hline+2 years & $19 / 35(54 \%)$ & $11 / 22(54 \%)$ & $6 / 16(37 \%)$ & $7 / 10(70 \%)$ & $43 / 83(52 \%)$ \\
\hline+3 years & $14 / 32(44 \%)$ & $7 / 19(37 \%)$ & $1 / 8(11 \%)$ & $2 / 9(22 \%)$ & $24 / 68(35 \%)$ \\
\hline+4 years & $9 / 29(31 \%)$ & $4 / 19(21 \%)$ & $0 / 5(0 \%)$ & $3 / 8(37 \%)$ & $16 / 61(26 \%)$ \\
\hline+5 years & 4/18 (22\%) & 2/18 (11\%) & $0 / 3(0 \%)$ & $1 / 4$ (25\%) & $7 / 43(16 \%)$ \\
\hline+6 years & $3 / 13(23 \%)$ & 2/17 (12\%) & $0 / 1(0 \%)$ & 1/3 (33\%) & 6/34 (18\%) \\
\hline+7 years & $2 / 5(40 \%)$ & $3 / 15(20 \%)$ & $0 / 1(0 \%)$ & $1 / 1(100 \%)$ & $6 / 22(27 \%)$ \\
\hline+8 years & $0 / 2(0 \%)$ & 2/14 (14\%) & $0 / 1(0 \%)$ & $0 / 0$ & 2/17 (12\%) \\
\hline+9 years & $0 / 0$ & 1/9 (11\%) & $0 / 1(0 \%)$ & $0 / 0$ & $1 / 10(10 \%)$ \\
\hline
\end{tabular}

$10 \pm 9$ months (range 1 month -5 years), the mean disappearence was at $16 \pm 4$ months (range 1 month-6 years), and the recurrence of MC was observed in $17 \%$ of cases, $29 \pm 23$ months (range 4 months-9 years) after MC disappearence. Recurrence was observed in 4/37 kidney and whole pancreas patients, 8/ 20 kidney and segmental pancreas patients, 1/16 kidney patients, $1 / 8$ kidney and islets patients. We detected MC more often in patients who had previously experienced an acute renal rejection or CMV infection or both: acute rejection was $61 \%$ compared with $39 \%$ non-acute rejection $(p=0.01)$; CMV infection was $56 \%$ compared with $44 \%$ non-CMV infection ( $p=\mathrm{ns})$. CMV infection and acute rejection occurring in the same patient further increased the risk of developing MC: rejection plus CMV infection was $89 \%$ compared with $42 \%$ non-rejection plus non-CMV infection $(p=0.01)$. No significant difference in MC incidence was found between the patients who experienced a course of OKT3 treatment and those who did not $(84 \%$ vs $81 \%, p=n s)$. Most patients showed one to three MC without further haematological abnormalities. In two patients five and six MC were detected. Both patients developed a beta-cell lymphoma (PTLD) shortly after kidney and whole pancreas transplantation. The first patient was a 45 years old and was treated for three kidney rejection episodes with steroid pulses (3 days) and OKT3 (14 days). He developed fever on day 44 , and his general condition deteriorated. On day 54 five MC were detected. On day 60 , hepatic failure ensued. He developed intravascular disseminated coagulation and died 5 days later. At autopsy PTLD affected (listed in decreasing intensity); allograft and native kidney and pancreas, liver, duodenum and antrum, spleen, heart and brain. This patient was serologically positive for EBV at the time of Tx, with no serologic evidence of subsequent EBV infection. The second patient was a 34 years old and underwent two acute rejection episodes, treated with three boluses of steroids, OKT3 (18 days) and ATG (10 days). He developed fever and an enlargment of cervical and axillary lymph nodes. On day 47 , six MC were detected. The histological examination of an axillary lymph node and liver biopsy confirmed the diagnosis of PTLD, positive for EBV. All immunosuppression was interrupted. The clinical picture ameliorated progressively with disappearance of lymphoadenopathy. Two MC were detectable at 6 months, and disappeared at 9 months. This patient is still alive 5 years after transplantation, with both transplanted organs functioning.

Before transplantation EBV and CMV serology (IgG positive or negative) transplantation did not influence the rate of MC (Table 3). CMV seroconversion or reactivation during the follow-up was observed in $30 \%$ of patients who developed MC and in $24 \%$ of patients who did not develop MC. EBV seroconversion or reactivation during the follow-up was observed in $14 \%$ of patients who developed MC and in $7 \%$ of patients who did not develop MC. 
Table 3. No difference between CMV and EBV serology (IgG positivity) before transplantation and during the study in patients who developed MC (MC Pos), compared with patients who did not develop MC (MC Neg)

\begin{tabular}{lcll}
\hline Serology before transplantation & MC Pos & MC Neg \\
\hline CMV Pos & $80 \%$ & $81 \%$ & $19 \%$ \\
CMV Neg & $20 \%$ & $83 \%$ & $17 \%$ \\
EBV Pos & $91 \%$ & $82 \%$ & $18 \%$ \\
EBV Neg & $19 \%$ & $71 \%$ & $29 \%$ \\
\hline
\end{tabular}

\section{Discussion}

The detection of MC in our patients was more frequent than reported previously in kidney graft recipients. The higher incidence of immunoglobulin abnormalities in patients on cyclosporin has been described $[14,15,16]$. Cyclosporin interferes with T-cell function blocking the release of interleukin 2; impaired control of beta-cell clones by suppressor T-cells could predispose to the development of MC. Only in a minority of cases does this clonal expansion progress to malignant PTLD $[13,15]$. We gave cyclosporin and ALG/ATG to all patients, as is included in our standard anti-rejection prophylactic regimen which could explain our high incidence of MC.

As in previous reports [13-16], MC in our patients were first detected within 1 year of transplantation. They were mostly transient which with the low concentration of the bands, suggests their benign nature.

The two patients who developed a beta-cell lymphoma showed five and six MC on immunofixation, respectively, shortly before neoplasms were diagnosed. In both patients overimmunosuppression resulted from treatment of steroid-resistant rejection episodes, after initial MC detection. Moreover, association with an Epstein-Barr virus infection was demonstrated, which by activating beta-cells, can induce their transformation especially when reduced T-cell surveillance is present. Only these two patients developed a number of MC greater than four. These findings should warn of the danger of malignant evolution when a policlonal escape occurs underlying a major immunological disorder. When a clone takes advantage and grows uncontrolled a lymphoproliferative disease is the final consequence. Immunosuppression withdrawal could be life-saving in these cases, especially before the malignant transformation - helping to restore the T-suppressor control.

The higher incidence of MC in combined kidney and pancreas than in kidney, and kidney plus islets recipients could be due to the higher rate of kidney rejection in these patients, leading to higher immunosuppression. Surprisingly, patients who received OKT3 after methylprednisolone for steroid-resistant rejections did not show an increased incidence of MC. No difference in viral infections was found among the different groups of patients.
In our study we did not find any correlation between CMV and EBV serology before transplantation and the development of MC. Both acute rejection and cytomegalovirus infection correlated with $\mathrm{MC}$ detection. Development of MC during or after cytomegalovirus infection is known to occur in groups who have and who have not received transplants [17].

In conclusion kidney and pancreas recipients, experiencing both cytomegalovirus infection and acute rejection, are at greatest risk of developing MC. Such MC appear to be benign and transient. Multiple bands detection may be a marker for subsequent progression to PTLD, in particular when associated with overimmunosuppression or Epstein Barr virus infection or both.

\section{References}

1. Secchi A, Di Carlo V, Martinenghi S et al. (1991) Effect of pancreas transplantation on life expectancy, kidney function and quality of life in uraemic Type 1 (insulin-dependent) diabetic patients. Diabetologia 34 [Suppl 1]: S141-S144

2. Sutherland DER, Najarian JS, Greenberg BZ et al. (1981) Hormonal and metabolic effects of a pancreatic endocrine graft. Ann Intern Med 95: 537-541

3. Solders G, Tyden G, Persson A, Groth C (1991) Improvement in diabetic neuropathy 4 years after successful pancreatic and renal transplantation. Diabetologia 34 [Suppl 1]: S125-S127

4. Richards KF, Belnap LeGP, Rees WV, Stevens LE (1989) Increased incidence of kidney rejection episodes in patients receiving combined kidney-pancreas transplant. Diabetes 38 [Suppl 1]: 251-252

5. Penn I (1979) Tumour incidence in human allograft recipients. Transpl Proc 11: 1047-1051

6. Penn I (1991) The changing pattern of posttransplant malignancies. Transpl Proc 23: 1101-1103

7. Sheil AGR, Flavel S, Disney APS, Matthew TH (1985) Cancer in dialysis and transplant patients. Transplant Proc 17: 195-198

8. Gruber SA, Chan GLC, Canafax DM, Matas AJ (1991) Immunosuppression in renal transplantation I. Cyclosporine and azathioprine. Clin Transplant 5: 65-85

9. Gruber SA, Chan GLC, Canafax DM, Matas AJ (1991) Immunosuppression in renal transplantation II. Corticosteroids, antilymphocyte globulin and OKT3. Clin Transplant 5: 219-232

10. Martinenghi S, Dell'Antonio G, Secchi A, Di Carlo V, Pozza G (1997) Cancer arising after pancreas and/or kidney transplantation in a series of 99 diabetic patients. Diabetes Care 20 (3): 272-275

11. Starzl TE, Porter KA, Iwatsuki S et al. (1984) Reversibility of lymphomas and lymphoproliferative lesions developing under cyclosporin-steroid therapy. Lancet 324: 583-587

12. Axelsson U, Bachman R, Hallen J (1966) Frequency of pathological proteins (M-components) in 6,995 sera from an adult population. Acta Med Scand 179: 235-247

13. Passweg J, Thiel G, Bock HA (1996 ) Monoclonal gammopathy after intense induction immunosuppression in renal transplant patients. Nephrol Dial Transplant 11: 2461-2465

14. Radl J, Valentijn RM, Haaijman JJ, Paul LC (1985) Monoclonal gammapathies in patients undergoing immunosuppressive treatment after renal transplantation. Clinical immunology and immunopathology 37: 98-102

15. Stanko CK, Jeffery JR, Rush DN (1989) Monoclonal and multiclonal gammopathies after renal transplantation. Transplant Proc 21: 3330-3332

16. Touraine JL, El Yafi S, Bosi E et al. (1983) Immunoglobulin abnormalities and infectious lymphoproliferative syndrome in cyclosporine-treated transplant patients. Transplant Proc 15 [Suppl 1]: 2798-2804

17. Deteix P, Chapuis-Cellier C, Ghais Z et al. (1985) Systematic survey of immunoglobulin abnormalities; frequency and evolution in organ transplant recipients. Transplant Proc 17: 2651-2654 